\title{
COMPOSITION, NUMERICAL RANGE AND ARON-BERNER EXTENSION
}

\author{
YUN SUNG CHOI, DOMINGO GARCÍA, SUNG GUEN KIM and MANUEL MAESTRE*
}

\begin{abstract}
Given an entire mapping $f \in \mathscr{H}_{b}(X, X)$ of bounded type from a Banach space $X$ into $X$, we denote by $\bar{f}$ the Aron-Berner extension of $f$ to the bidual $X^{* *}$ of $X$. We show that $\overline{g \circ f}=\bar{g} \circ \bar{f}$ for all $f, g \in \mathscr{H}_{b}(X, X)$ if $X$ is symmetrically regular. We also give a counterexample on $l_{1}$ such that the equality does not hold. We prove that the closure of the numerical range of $f$ is the same as that of $\bar{f}$.
\end{abstract}

\section{Introduction}

Given complex Banach spaces $X$ and $Y$, we denote by $\mathscr{P}\left({ }^{n} X, Y\right)$ the Banach space of bounded $n$-homogeneous polynomials of $X$ into $Y$. When $Y$ is the scalar field $\mathrm{C}$, we denote this space by $\mathscr{P}\left({ }^{n} X\right)$. We recall that a bounded $n$-homogeneous polynomial $P \in \mathscr{P}\left({ }^{n} X, Y\right)$ is the restriction to the diagonal of a continuous $n$-linear mapping $A$ from $X$ into $Y$, that is, $P(x)=$ $A(x, \ldots, x), x \in X$. Each such $P$ has a unique associated bounded symmetric $n$-linear mapping $A$ from $X$ into $Y$. Each bounded $n$-homogeneous polynomial $P$ has a canonical extension $\bar{P} \in \mathscr{P}\left({ }^{n} X^{* *}, Y^{* *}\right)$ to the bidual $X^{* *}$ of $X$, which is called the Aron-Berner extension of $P$ ([2]) (see the next section for definitions). By [10, Theorem 3] (see also [2]), every entire mapping $f \in \mathscr{H}_{b}(X, Y)$ of bounded type extends in a canonical fashion to a mapping $\bar{f} \in \mathscr{H}_{b}\left(X^{* *}, Y^{* *}\right)$ in the following way. Given the Taylor series expansion of $f$ at $0, f=\sum_{n=0}^{\infty} P_{n}, \bar{f}$ is defined as $\bar{f}=\sum_{n=0}^{\infty} \overline{P_{n}}$.

Our first interest in this paper is to verify if $\frac{n=0}{g \circ f}=\bar{g} \circ \bar{f}$ for $f \in \mathscr{H}_{b}(X, Y)$ and $g \in \mathscr{H}_{b}(Y, Z)$. We are motivated by the following two problems: We consider the case $X=Y=Z$.

(1) The Aron-Berner extension is an isomorphism of the Fréchet space $\mathscr{H}_{b}(X, X)$ into the Fréchet space $\mathscr{H}_{b}\left(X^{* *}, X^{* *}\right)$ and both spaces are Fréchet

\footnotetext{
* The first named author's research is supported in part by KOSEF Interdisciplinary Research Program Grant 1999-2-102-003-5 of Korea. The second and fourth authors were supported by MEC and FEDER Project MTM2005-08210. The third author wishes to acknowledge the financial support of Korea Research Foundation Grant (KRF-2002-070-C00006).
}

Received January 30, 2007. 
algebras under composition. Is it true that the Aron-Berner extension is an isomorphism into between Fréchet algebras?

(2) Given $g \in \mathscr{H}_{b}(X, X)$ consider the composition operator $\varphi_{g}: \mathscr{H}_{b}(X, X)$ $\rightarrow \mathscr{H}_{b}(X, X)$ defined by $\varphi_{g}(f)=g \circ f$. This composition operator $\varphi_{g}$ is extended to the composition operator $\varphi_{\bar{g}}: \mathscr{H}_{b}\left(X^{* *}, X^{* *}\right) \rightarrow \mathscr{H}_{b}\left(X^{* *}, X^{* *}\right)$. Does the following diagram commute?

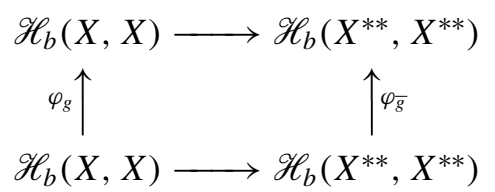

The answer to our questions is, in general, negative. In Section 2 we show the existence of a 2-homogeneous continuous polynomial $P: \ell_{1} \longrightarrow \ell_{1}$ such that $\overline{P \circ P} \neq \bar{P} \circ \bar{P}$.

Our second interest is to know if the Aron-Berner extension preserves numerical ranges. Lumer in 1961 ([14]) gave a theory of numerical range for bounded linear operators on Banach spaces. Harris in 1971 ([13]) developed a theory of numerical range and numerical radius for a holomorphic mapping. This theory has many applications. For example, he obtained an inequality ([13, Theorem 1]) which is a bound for each of the terms of the Taylor series expansion of a holomorphic mapping in terms of the numerical radius of the mapping. This inequality implies some results concerning the spectrum of holomorphic mappings ([13, Proposition 5]), the rotundity at the identity of the sup norm on holomorphic mappings ([12, Theorem 2]) and the extremal case of the Schwarz lemma ([11, Theorem 1]). We prove that the closure of the numerical range of $f \in \mathscr{H}_{b}(X, X)$ is the same as that of $\bar{f} \in \mathscr{H}_{b}\left(X^{* *}, X^{* *}\right)$, which implies that the numerical radius of $f$ is the same as that of $\bar{f}$.

\section{Aron-Berner extension and composition}

A bounded $n$-homogeneous polynomial $P \in \mathscr{P}\left({ }^{n} X, Y\right)$ has an extension $\bar{P} \in$ $\mathscr{P}\left({ }^{n} X^{* *}, Y^{* *}\right)$ to the bidual $X^{* *}$ of $X$, which is called the Aron-Berner extension of $P$. In fact, $\bar{P}$ is defined in the following way. Let $A$ be the symmetric $n$ linear mapping associated to $P, A$ can be extended to an $n$-linear mapping $\bar{A}$ from $X^{* *}$ into $Y^{* *}$ in such a way that for each fixed $j, 1 \leq j \leq n$, and for each fixed $x_{1}, \ldots, x_{j-1} \in X$ and $z_{j+1}, \ldots, z_{m} \in X^{* *}$, the linear mapping

$$
z \rightarrow \bar{A}\left(x_{1}, \ldots, x_{j-1}, z, z_{j+1}, \ldots, z_{n}\right), \quad z \in X^{* *},
$$

is $\left(w^{*}, w^{*}\right)$-continuous. In other words, we define $\bar{A}\left(x_{1}, \ldots, x_{j-1}, z, z_{j+1}\right.$, $\left.\ldots, z_{n}\right)$ to be the weak-star limit of the net $\left(\bar{A}\left(x_{1}, \ldots, x_{j-1}, x_{\alpha}, z_{j+1}, \ldots, z_{n}\right)\right)$ for a weak-star convergent net $\left(x_{\alpha}\right) \subset X$ to $z$. By this $\left(w^{*}, w^{*}\right)$-continuity $A$ 
can be extended to an $n$-linear mapping $\bar{A}$ from $X^{* *}$ into $Y^{* *}$, beginning with the last variable and working backwards to the first. Then the restriction

$$
\bar{P}(z)=\bar{A}(z, \ldots, z)
$$

is called the Aron-Berner extension of $P$. Given $z \in X^{* *}$ and $w \in Y^{*}$, we have

$$
\bar{P}(z)(w)=\overline{w \circ P}(z) .
$$

Actually this equality is often used as the definition of the vector-valued AronBerner extension based upon the scalar-valued Aron-Berner extension. Davie and Gamelin [10, Theorem 8] proved that $\|P\|=\|\bar{P}\|$. It is also worth remarking that $\bar{A}$ is not symmetric in general.

A complex Banach space $X$ is called symmetrically regular if every continuous symmetric linear mapping $T: X \rightarrow X^{*}$ is weakly compact. Recall that $T$ is symmetric means that $T(x)(y)=T(y)(x)$ for all $x, y \in X$. If $X$ is symmetrically regular then, by $[3,8.3$ Theorem], $\bar{A}$ is also symmetric and separately weak-star continuous on $X^{* *}$, for all symmetric $n$-linear form $A: X \times \cdots \times X \rightarrow \mathrm{C}$.

THeOREM 2.1. Let $X, Y$ and $Z$ be complex Banach spaces. If $Y$ is symmetrically regular then $\overline{Q \circ\left(P_{0}+P_{1}+\cdots+P_{m}\right)}=\bar{Q} \circ\left(P_{0}+\overline{P_{1}}+\cdots+\overline{P_{m}}\right)$ for every $P_{i} \in \mathscr{P}\left({ }^{i} X, Y\right)$, for $i=0,1, \ldots, m, Q \in \mathscr{P}\left({ }^{k} Y, Z\right)$ and $m, k \geq 1$.

Proof. Let us denote $P=P_{0}+P_{1}+\ldots+P_{m}$, and let $B$ be the symmetric $k$-linear form associated to $Q$. We put $\mathscr{J}=\left\{\mathbf{j}=\left(j_{0}, \ldots, j_{m}\right) \mid \sum_{h=0}^{m} j_{h}=\right.$ $\left.k, 0 \leq j_{h} \leq k, h=0,1, \ldots, m\right\}$ and $|\mathbf{j}|=\sum_{h=0}^{m} h j_{h}$. We have

$$
Q \circ P(x)=\sum_{\left(j_{0}, \ldots, j_{m}\right) \in \mathscr{J}}\left(\begin{array}{c}
k \\
j_{0}, \ldots, j_{m}
\end{array}\right) B\left(P_{0}^{j_{0}}, P_{1}^{j_{1}}(x), \ldots, P_{m}^{j_{m}}(x)\right),
$$

for all $x \in X$, where $P_{i}^{j_{i}}$ means that the coordinate $P_{i}$ is repeated $j_{i}$-times. The mapping $R_{\mathbf{j}}(x)=B\left(P_{0}^{j_{0}}, P_{1}^{j_{1}}(x), \ldots, P_{m}^{j_{m}}(x)\right)$ is a continuous $|\mathbf{j}|$-homogeneous polynomial on $X$ for all $\mathbf{j} \in \mathscr{J}$. Hence

$$
\overline{Q \circ P}(z)=\sum_{\mathbf{j}=\left(j_{0}, \ldots, j_{m}\right) \in \mathscr{F}}\left(\begin{array}{c}
k \\
j_{0}, \ldots, j_{m}
\end{array}\right) \overline{R_{\mathbf{j}}}(z),
$$

for all $z \in X^{* *}$. On the other hand, as $Y$ is symmetrically regular, $\bar{B}$ is symmetric and hence

$$
\bar{Q} \circ \bar{P}(z)=\sum_{\mathbf{j}=\left(j_{0}, \ldots, j_{m}\right) \in \mathscr{J}}\left(\begin{array}{c}
k \\
j_{0}, \ldots, j_{m}
\end{array}\right) T_{\mathbf{j}}(z),
$$


where $T_{\mathbf{j}}(z)=\bar{B}\left(P_{0}^{j_{0}},{\overline{P_{1}}}^{j_{1}}(z), \ldots,{\overline{P_{m}}}^{j_{m}}(z)\right)$ for all $z \in X^{* *}$. If we prove that $\overline{R_{\mathbf{j}}}=T_{\mathbf{j}}$ for all $\mathbf{j} \in \mathscr{J}$ with $|\mathbf{j}|>0$, then $\overline{Q \circ P}=\bar{Q} \circ \bar{P}$.

Recall that the differential of a polynomial $P \in \mathscr{P}\left({ }^{k} X, Y\right)$ is the $(k-1)$ homogeneous polynomial $D(P): X \rightarrow \mathscr{L}(X, Y)$ given by $D(P)(x)(z)=$ $k A(x, \ldots, x, z),(x, z \in X)$, where $A$ is the symmetric $k$-linear mapping associated to $P$.

Given $\mathbf{j} \in \mathscr{J}$ with $|\mathbf{j}|>0$, we have $R_{\mathbf{j}}(x)=T_{\mathbf{j}}(x)$ for all $x \in X$, hence, by [7, Proposition 1.1] (see also [15, Theorem 2]), $\overline{R_{\mathbf{j}}}=T_{\mathbf{j}}$ if and only if the following two properties hold:

(a) For every $x \in X, D\left(T_{\mathbf{j}}\right)(x): X^{* *} \rightarrow Z^{* *}$ is $\left(w^{*}, w^{*}\right)$-continuous.

(b) For every $z \in X^{* *}$ and every net $\left(x_{\mu}\right) \subset X$ such that $\left(x_{\mu}\right)$ converges weak-star to $z, D\left(T_{\mathbf{j}}\right)(z)\left(x_{\mu}\right)$ converges weak-star to $D\left(T_{\mathbf{j}}\right)(z)(z)$ in $Z^{* *}$.

We consider $C_{\mathbf{j}}: X^{* *} \longrightarrow Y^{* *}$ the bounded $|\mathbf{j}|$-linear mapping defined by

$$
\begin{aligned}
& C_{\mathbf{j}}\left(z_{1}, \ldots, z_{|\mathbf{j}|}\right) \\
& =\bar{B}\left(P_{0}^{j_{0}}, \overline{A_{1}}\left(z_{1}\right), \ldots, \overline{A_{1}}\left(z_{j_{1}}\right), \overline{A_{2}}\left(z_{j_{1}+1}, z_{j_{1}+2}\right), \ldots, \overline{A_{2}}\left(z_{j_{1}+2 j_{2}-1}, z_{j_{1}+2 j_{2}}\right),\right. \\
& \left.\quad \ldots, \overline{A_{m}}\left(z_{\sum_{h=1}^{m-1} h j_{h}+1}, \ldots, z_{\sum_{h=1}^{m-1} h j_{h}+m}\right), \ldots, \overline{A_{m}}\left(z_{|\mathbf{j}|-m+1}, \ldots, z_{|\mathbf{j}|} \mid\right)\right),
\end{aligned}
$$

where $A_{h}$ is the symmetric $h$-linear mapping associated to $P_{h}$ for $h=1, \ldots, m$. Clearly $T_{\mathbf{j}}(z)=C_{\mathbf{j}}(z, \ldots, z)$ for all $z \in X^{* *}$. If $S C_{\mathbf{j}}$ denotes the symmetrization of $C_{\mathbf{j}}$, we have that

$$
S C_{\mathbf{j}}\left(z_{1}, \ldots, z_{|\mathbf{j}|}\right)=\frac{1}{|\mathbf{j}| !} \sum_{\sigma \in S_{|\mathbf{j}|}} C_{\sigma \mathbf{j}}\left(z_{1}, \ldots, z_{|\mathbf{j}|}\right),
$$

where $\mathbf{S}_{|\mathbf{j}|}$ stands for the group of permutations of $\{1,2, \ldots,|\mathbf{j}|\}$ and

$$
C_{\sigma \mathbf{j}}\left(z_{1}, \ldots, z_{|\mathbf{j}|}\right)=C_{\mathbf{j}}\left(z_{\sigma(1)}, \ldots, z_{\sigma(|\mathbf{j}|)}\right) .
$$

With this notation

$$
D\left(T_{\mathbf{j}}\right)(z)(w)=|\mathbf{j}| S C_{\mathbf{j}}(z, \ldots, z, w)=\frac{1}{(|\mathbf{j}|-1) !} \sum_{\sigma \in S_{|\mathbf{j}|}} C_{\sigma \mathbf{j}}(z, \ldots, z, w),
$$

for all $z, w \in X^{* *}$.

We know that $\bar{B}$ is symmetric. On the other hand

$$
\overline{A_{h}}(z, \ldots, z, x)=\overline{A_{h}}(z, \ldots, z, x, z)=\cdots=\overline{A_{h}}(x, z, \ldots, z)
$$


for all $z \in X^{* *}, x \in X$ and $h=1, \ldots, m$. Thus, for fixed $\sigma \in \mathbf{S}_{|\mathbf{j}|}$ there exists a unique $h=1, \ldots, m$ such that

$$
\begin{aligned}
C_{\sigma \mathbf{j}}(z, \ldots, z, x)=\bar{B}\left(P_{0}^{j_{0}},{\overline{P_{1}}}^{j_{1}}(z), \ldots,{\overline{P_{h-1}}}^{j_{h-1}}(z),\right. \\
\left.\overline{A_{h}}(x, z, \ldots, z),{\overline{P_{h+1}}}^{j_{h+1}}(z), \ldots,{\overline{P_{m}}}^{j_{m}}(z)\right) .
\end{aligned}
$$

The linear mapping $\overline{A_{h}}(-, z \ldots, z)$ is weak-star continuous on $X^{* *}$. Since $Y$ is symmetrically regular, $\bar{B}$ is weak-star separately continuous. Hence, if $\left(x_{\mu}\right) \subset X$ converges weak-star to $z$ in $X^{* *}$, then $C_{\sigma \mathbf{j}}\left(z, \ldots, z, x_{\mu}\right)$ converges weak-star to $T_{\mathbf{j}}(z)$. As an immediate consequence $D\left(T_{\mathbf{j}}\right)(z)\left(x_{\mu}\right)$ converges to $|\mathbf{j}| T_{\mathbf{j}}(z)=D\left(T_{\mathbf{j}}\right)(z)(z)$ for all $z \in X^{* *}$ and property (b) holds for every $T_{\mathbf{j}}$.

Finally, given $x \in X$ and $w \in X^{* *}$, we have $\overline{A_{h}}(x, \ldots, x, w)=$ $\overline{A_{h}}(x, \ldots, x, w, x)=\cdots=\overline{A_{h}}(w, x, \ldots, x)$ and the linear mapping $\overline{A_{h}}(x, \ldots, x,-)$ is weak-star continuous on $X^{* *}$ for all $h=1, \ldots, m$. As

$$
\begin{aligned}
& C_{\sigma \mathbf{j}}(x, \ldots, x, w)=\bar{B}\left(P_{0}^{j_{0}}, P_{1}^{j_{1}}(x), \ldots, P_{h-1}^{j_{h-1}}(x),\right. \\
&\left.\overline{A_{h}}(x, \ldots, x, w), P_{h+1}^{j_{h+1}}(x), \ldots, P_{m}^{j_{m}}(x)\right),
\end{aligned}
$$

the proof that property (a) holds for every $T_{\mathbf{j}}$ can be obtained in a similar way.

Corollary 2.2. Suppose that $Y$ is symmetrically regular. Then $\overline{g \circ f}=$ $\bar{g} \circ \bar{f}$ for $f \in \mathscr{H}_{b}(X, Y)$ and $g \in \mathscr{H}_{b}(Y, Z)$.

Proof. We first note that the Taylor series $\sum_{n=0}^{\infty} Q_{n}$ of $g$ at 0 converges to $g$ in the Fréchet space $\mathscr{H}_{b}(Y, Z)$. Since the Aron-Berner extension induces a Fréchet isomorphism from $\mathscr{H}_{b}(Y, Z)$ into $\mathscr{H}_{b}\left(Y^{* *}, Z^{* *}\right)$, it is enough to consider only the case where $g=Q \in \mathscr{P}\left({ }^{k} Y, Z\right)$, for all $k \geq 1$.

For $R>0$ we consider on $\mathscr{H}_{b}(X, Y)$ the norm $\|f\|_{R}=\sup \{|f(x)|:\|x\| \leq$ $R\}$. We fix $Q \in \mathscr{P}\left({ }^{k} Y, Z\right)$ and $f \in \mathscr{H}_{b}(X, Y)$. There exists $S>0$ such that $f\left(R B_{X}\right) \subset S B_{Y}$. Since $Q$ is uniformly continuous on the ball $(S+1) B_{Y}$ and since $\bar{Q}$ is also uniformly continuous on $(S+1) B_{Y^{* *}}$, given $\varepsilon>0$ we can find $0<\delta<1$ such that $\left\|Q\left(y_{1}\right)-Q\left(y_{2}\right)\right\|<\varepsilon$ for all $y_{1}, y_{2} \in(S+1) B_{Y}$ with $\left\|y_{1}-y_{2}\right\|<\delta$ and $\left\|\bar{Q}\left(v_{1}\right)-\bar{Q}\left(v_{2}\right)\right\|<\varepsilon$ for all $v_{1}, v_{2} \in(S+1) B_{Y^{* *}}$ with $\left\|v_{1}-v_{2}\right\|<\delta$.

The Taylor series expansion $\sum_{m=0}^{\infty} P_{m}$ of $f$ at zero converges absolutely and uniformly to $f$ on any bounded set of $X$, and hence there exists $m_{0}$ such that

$$
\left\|f-\sum_{m=0}^{m_{0}} P_{m}\right\|_{R}<\delta .
$$


Thus, $\left\|Q \circ f-Q \circ\left(\sum_{m=0}^{m_{0}} P_{m}\right)\right\|_{R}<\varepsilon$. Hence, by [10, Theorem 8],

$$
\left\|\overline{Q \circ f}-\overline{Q \circ\left(\sum_{m=0}^{m_{0}} P_{m}\right)}\right\|_{R}=\left\|Q \circ f-Q \circ\left(\sum_{m=0}^{m_{0}} P_{m}\right)\right\|_{R}<\varepsilon,
$$

which, by Theorem 2.1, implies

$$
\left\|\overline{Q \circ f}-\bar{Q} \circ\left(\sum_{m=0}^{m_{0}} \overline{P_{m}}\right)\right\|_{R}=\left\|\overline{Q \circ f}-\overline{Q \circ\left(\sum_{m=0}^{m_{0}} P_{m}\right)}\right\|_{R}<\varepsilon
$$

On the other hand, by (1) and [10, Theorem 8] we have $\left\|\bar{f}-\sum_{m=0}^{m_{0}} \overline{P_{m}}\right\|_{R}=$ $\left\|f-\sum_{m=0}^{m_{0}} P_{m}\right\|_{R}<\delta$, from which

$$
\left\|\bar{Q} \circ \bar{f}-\bar{Q} \circ\left(\sum_{m=0}^{m_{0}} \overline{P_{m}}\right)\right\|_{R}<\varepsilon .
$$

Now the conclusion is clear from (2) and (3).

An $f \in \mathscr{H}_{b}(X, Y)$ is called weakly compact if $f\left(r B_{X}\right)$ is a relatively weakly compact set for all $r>0$. Let $\sum_{m=0}^{\infty} P_{m}$ be the Taylor series expansion of $f$ at zero. An obvious modification of [4, Proposition 3.4] shows that $f$ is weakly compact if and only if $P_{m}\left(B_{X}\right)$ is a relatively weakly compact set for all $m=1,2, \ldots$.

Proposition 2.3. Let $X, Y$ and $Z$ be complex Banach spaces and $m \geq 1$. If $P_{h} \in \mathscr{P}\left({ }^{h} X, Y\right)$ is a weakly compact polynomial for all $h=1, \ldots, m$ and $P=\sum_{h=0}^{m} P_{h}$, then $\overline{Q \circ P}=\bar{Q} \circ \bar{P}$ for every $Q \in \mathscr{P}\left({ }^{k} Y, Z\right)$ and $k \geq 1$.

Proof. Let $B$ be the $k$-linear symmetric mapping associated to $Q$ and $\bar{B}$ be its Aron-Berner extension. An inspection of the proof of Theorem 2.1 shows that the symmetry of $\bar{B}$ on $\left(\operatorname{span}\left(\bar{P}\left(X^{* *}\right)\right)^{k}\right.$ is a sufficient condition for the equality $\overline{Q \circ P}=\bar{Q} \circ \bar{P}$. Since $\bar{P}\left(X^{* *}\right)=P(X) \subset Y$, the conclusion follows.

It is well-known that the Banach space $l_{1}$ is not symmetrically regular ([3]). In the following we construct a 2-homogeneous polynomial $P: l_{1} \rightarrow l_{1}$ such that $\overline{P \circ P} \neq \bar{P} \circ \bar{P}$. 
Example 2.4. Define the bounded symmetric bilinear mappings $A_{1}, A_{2}$ : $l_{1} \times l_{1} \rightarrow l_{1}$ by

$$
\begin{gathered}
A_{1}(x, y)=\sum_{n=1}^{\infty}\left[\left(x_{1} e_{1}+x_{3} e_{3}+\cdots+x_{2 n-1} e_{2 n-1}\right) y_{2 n}\right. \\
\left.+\left(y_{1} e_{1}+y_{3} e_{3}+\cdots+y_{2 n-1} e_{2 n-1}\right) x_{2 n}\right], \\
A_{2}(x, y)=\sum_{n=1}^{\infty}\left[\left(x_{1}+x_{3}+\cdots+x_{2 n-1}\right) y_{2 n}\right. \\
\left.+\left(y_{1}+y_{3}+\cdots+y_{2 n-1}\right) x_{2 n}\right] e_{2 n},
\end{gathered}
$$

where $x=\left(x_{i}\right), y=\left(y_{i}\right) \in l_{1}$ and $\left\{e_{n}\right\}$ is the canonical basis of $l_{1}$. Let $A=A_{1}+A_{2}$.

Let $P$ be the 2-homogeneous polynomial from $l_{1}$ to $l_{1}$ associated to $A$. Then $\overline{P \circ P} \neq \bar{P} \circ \bar{P}$.

Proof. We can see easily that

$$
\begin{array}{ll}
A_{1}\left(e_{2 p}, e_{2 q}\right)=0, & A_{1}\left(e_{2 p-1}, e_{2 q-1}\right)=0, \\
A_{2}\left(e_{2 p}, e_{2 q}\right)=0, & A_{2}\left(e_{2 p-1}, e_{2 q-1}\right)=0
\end{array}
$$

for every positive integers $p, q$. Further, we obtain that

$$
A_{1}\left(e_{2 p}, e_{2 q-1}\right)= \begin{cases}e_{2 q-1} & \text { if } p \geq q, \\ 0 & \text { if } p<q\end{cases}
$$

and

$$
A_{2}\left(e_{2 p}, e_{2 q-1}\right)= \begin{cases}e_{2 p} & \text { if } p \geq q \\ 0 & \text { if } p<q .\end{cases}
$$

Let $\alpha$ and $\beta$ be weak-star limit points in $\ell_{1}^{* *} \backslash \ell_{1}$ of the sets $\left\{e_{2 k-1}: k \in \mathbf{N}\right\}$ and $\left\{e_{2 k}: k \in \mathrm{N}\right\}$, respectively. It follows immediately from the above that

$$
\begin{aligned}
& \overline{A_{1}}\left(e_{2 q-1}, \alpha\right)=\overline{A_{1}}\left(e_{2 p}, \alpha\right)=\overline{A_{1}}\left(e_{2 p}, \beta\right)=0, \\
& \overline{A_{2}}\left(e_{2 q-1}, \alpha\right)=\overline{A_{2}}\left(e_{2 p}, \alpha\right)=\overline{A_{2}}\left(e_{2 p}, \beta\right)=0, \\
& \overline{A_{1}}\left(e_{2 q-1}, \beta\right)=e_{2 q-1}, \\
& \overline{A_{2}}\left(e_{2 q-1}, \beta\right)=\beta
\end{aligned}
$$


for every positive integers $p$ and $q$. By taking limits we have that

$$
\begin{aligned}
& \overline{A_{1}}(\alpha, \alpha)=\overline{A_{1}}(\beta, \alpha)=\overline{A_{1}}(\beta, \beta)=0, \\
& \overline{A_{2}}(\alpha, \alpha)=\overline{A_{2}}(\beta, \alpha)=\overline{A_{2}}(\beta, \beta)=0, \\
& \overline{A_{1}}(\alpha, \beta)=\alpha, \\
& \overline{A_{2}}(\alpha, \beta)=\beta,
\end{aligned}
$$

which implies that

$$
\bar{A}(\alpha, \alpha)=\bar{A}(\beta, \beta)=\bar{A}(\beta, \alpha)=0, \quad \bar{A}(\alpha, \beta)=\alpha+\beta .
$$

A simple computation shows that

$$
\begin{aligned}
& \bar{P}(\alpha+\beta)=\bar{A}(\alpha+\beta, \alpha+\beta)=\alpha+\beta, \\
& \bar{A}\left(e_{2 q-1}+e_{2 p}, \alpha+\beta\right)=e_{2 q-1}+\beta,
\end{aligned}
$$

for every positive integers $p$ and $q$. Therefore, it is clear that $(\bar{P} \circ \bar{P})(\alpha+\beta)=$ $\bar{P}(\alpha+\beta)=\alpha+\beta$. However, it can be computed that $\overline{P \circ P}(\alpha+\beta)=\frac{5}{3}(\alpha+\beta)$. Indeed, let $\left(x_{\mu}\right)$ be a net in $X$ converging weak-star to $(\alpha+\beta)$ such that each $x_{\mu}$ is of the form $\left(e_{2 q-1}+e_{2 p}\right)$. Let $C$ be the bounded symmetric 4-linear mapping associated to $Q \circ P$. Then

$$
\begin{aligned}
C\left(x_{1}, x_{2}, x_{3}, x_{4}\right)=\frac{1}{3} & {\left[A\left(A\left(x_{1}, x_{2}\right), A\left(x_{3}, x_{4}\right)\right)\right.} \\
& \left.+A\left(A\left(x_{1}, x_{3}\right), A\left(x_{2}, x_{4}\right)\right)+A\left(A\left(x_{1}, x_{4}\right), A\left(x_{2}, x_{3}\right)\right)\right] .
\end{aligned}
$$

Let $x_{\mu}^{j}=x_{\mu}$ for $j=1,2,3,4$. We also write each form of $x_{\mu}^{j}$ as $\left(e_{2 q-1}^{j}+e_{2 p}^{j}\right)$ if necessary. Since $\left(x_{\mu}\right)$ converges weak-star to $\alpha+\beta$, we have

$$
\overline{P \circ P}(\alpha+\beta)=\left(w^{*}-\lim \right)_{x_{\mu}^{1}} \cdots\left(w^{*}-\lim \right)_{x_{\mu}^{4}} C\left(x_{\mu}^{1}, x_{\mu}^{2}, x_{\mu}^{3}, x_{\mu}^{4}\right) .
$$

The computation of the limit is as follows:

$$
\begin{aligned}
\left(w^{*}-\right. & \lim )_{x_{\mu}^{1}} \cdots\left(w^{*}-\lim \right)_{x_{\mu}^{4}} A\left(A\left(x_{\mu}^{1}, x_{\mu}^{2}\right), A\left(x_{\mu}^{3}, x_{\mu}^{4}\right)\right) \\
& =(\bar{P} \circ \bar{P})(\alpha+\beta) \\
& =\alpha+\beta
\end{aligned}
$$




$$
\begin{aligned}
\left(w^{*}-\right. & \lim )_{x_{\mu}^{1}} \cdots\left(w^{*}-\lim \right)_{x_{\mu}^{4}} A\left(A\left(x_{\mu}^{1}, x_{\mu}^{3}\right), A\left(x_{\mu}^{2}, x_{\mu}^{4}\right)\right) \\
& =\left(w^{*}-\lim \right)_{x_{\mu}^{1}}\left(w^{*}-\lim \right)_{x_{\mu}^{2}} \bar{A}\left(\bar{A}\left(x_{\mu}^{1}, \alpha+\beta\right), \bar{A}\left(x_{\mu}^{2}, \alpha+\beta\right)\right) \\
& =\left(w^{*}-\lim \right) x_{\mu}^{1}\left(w^{*}-\lim \right)_{x_{\mu}^{2}} \bar{A}\left(e_{2 q-1}^{1}+\beta, e_{2 q-1}^{2}+\beta\right) \\
& =2(\alpha+\beta),
\end{aligned}
$$

and

$$
\begin{aligned}
\left(w^{*}-\right. & \lim )_{x_{\mu}^{1}} \cdots\left(w^{*}-\lim \right)_{x_{\mu}^{4}} A\left(A\left(x_{\mu}^{1}, x_{\mu}^{4}\right), A\left(x_{\mu}^{2}, x_{\mu}^{3}\right)\right) \\
& =\left(w^{*}-\lim \right)_{x_{\mu}^{1}} \cdots\left(w^{*}-\lim \right)_{x_{\mu}^{4}} A\left(A\left(x_{\mu}^{2}, x_{\mu}^{3}\right), A\left(x_{\mu}^{1}, x_{\mu}^{4}\right)\right) \\
& =\left(w^{*}-\lim \right)_{x_{\mu}^{1}} \bar{A}\left(\bar{P}(\alpha+\beta), \bar{A}\left(x_{\mu}^{1}, \alpha+\beta\right)\right) \\
& =\left(w^{*}-\lim \right)_{x_{\mu}^{1}} \bar{A}\left(\alpha+\beta, e_{2 q-1}^{1}+\beta\right) \\
& =2(\alpha+\beta) .
\end{aligned}
$$

Therefore, $\overline{P \circ P}(\alpha+\beta)=\frac{5}{3}(\alpha+\beta)$.

The above example solves our main question in the negative, but the presentation given here is not our original point of view. Actually we found it by a more general mathematical tool, that is, the next lemma.

Lemma 2.5. Given two bounded 2-homogeneous polynomials $P \in \mathscr{P}\left({ }^{2} X, Y\right)$ and $Q \in \mathscr{P}\left({ }^{2} Y, Z\right)$, let $A$ and $B$ be the bounded symmetric bilinear mappings associated to $P$ and $Q$, respectively. Then

$$
\overline{Q \circ P}=\bar{Q} \circ \bar{P}
$$

if and only if $\bar{B}\left(\bar{P}(z), \bar{A}\left(x_{\mu}, z\right)\right)$ converges weak-star to $\bar{Q} \circ \bar{P}(z)$ for every net $\left(x_{\mu}\right) \subset X$ converging weak-star to $z \in X^{* *}$.

Proof. By [7, Proposition 1.1], $\overline{Q \circ P}=\bar{Q} \circ \bar{P}$ holds if and only if the properties (a) and (b) stated at the beginning of the proof of Theorem 2.1 hold. We have that $\bar{A}(x, z)=\bar{A}(z, x)$ for all $x \in X$ and $z \in X^{* *}$ and that $\bar{B}(y, u)=\bar{B}(u, y)$ for all $y \in Y$ and $u \in Y^{* *}$. Hence it is easily checked that the property (a) holds always.

The bilinear mapping $S \bar{A}: X^{* *} \times X^{* *} \longrightarrow Y^{* *}$ defined by $S \bar{A}\left(z_{1}, z_{2}\right)=$ $\frac{1}{2}\left(\bar{A}\left(z_{1}, z_{2}\right)+\bar{A}\left(z_{2}, z_{1}\right)\right)$ is the symmetrization of $\bar{A}$. If we consider $C$ : $\left(X^{* *}\right)^{4} \longrightarrow Z^{* *}$ defined by $C\left(z_{1}, z_{2}, z_{3}, z_{4}\right)=\bar{B}\left(S(\bar{A})\left(z_{1}, z_{2}\right), S(\bar{A})\left(z_{3}, z_{4}\right)\right)$ satisfies that $C(z, z, z, z)=\bar{Q} \circ \bar{P}(z)$ for all $z \in X^{* *}$. Hence the 4-linear symmetric mapping associated to $\bar{Q} \circ \bar{P}$ is $S C$, the symmetrization of $C$. A 
straightforward calculation gives

$$
\begin{aligned}
S C\left(z_{1}, z_{2}, z_{3}, z_{4}\right) \\
=\frac{1}{6}\left(\bar{B}\left(S \bar{A}\left(z_{1}, z_{2}\right), S \bar{A}\left(z_{3}, z_{4}\right)\right)+\bar{B}\left(S \bar{A}\left(z_{1}, z_{3}\right), S \bar{A}\left(z_{2}, z_{4}\right)\right)\right. \\
\quad+\bar{B}\left(S \bar{A}\left(z_{1}, z_{4}\right), S \bar{A}\left(z_{2}, z_{3}\right)\right)+\bar{B}\left(S \bar{A}\left(z_{2}, z_{3}\right), S \bar{A}\left(z_{1}, z_{4}\right)\right) \\
\left.\quad+\bar{B}\left(S \bar{A}\left(z_{2}, z_{4}\right), S \bar{A}\left(z_{1}, z_{3}\right)\right)+\bar{B}\left(S \bar{A}\left(z_{3}, z_{4}\right), S \bar{A}\left(z_{1}, z_{2}\right)\right)\right) .
\end{aligned}
$$

Hence

$$
\begin{aligned}
D(\bar{Q} \circ \bar{P})(z)(x) & =4 S C(z, z, z, x) \\
& =2 \bar{B}(\bar{P}(z), S \bar{A}(z, x))+2 \bar{B}(S \bar{A}(x, z), \bar{P}(z)),
\end{aligned}
$$

for all $x \in X$ and $z \in X^{* *}$. As $S \bar{A}(z, x)=S \bar{A}(x, z)=\bar{A}(x, z)$ for all $x \in X$ and $z \in X^{* *}$ we obtain that

$$
D(\bar{Q} \circ \bar{P})(z)(x)=2(\bar{B}(\bar{P}(z), \bar{A}(x, z))+\bar{B}(\bar{A}(x, z), \bar{P}(z))),
$$

for all $x \in X$ and $z \in X^{* *}$. The linear mappings $\bar{B}(-, \bar{P}(z))$ and $\bar{A}(-, z)$ are $\left(w^{\star}, w^{\star}\right)$-continuous. Hence, given a net $\left(x_{\mu}\right) \subset X$ converging weak-star to $z \in X^{* *}$ we have that the net $\left.\bar{B}\left(\bar{A}\left(x_{\mu}, z\right), \bar{P}(z)\right)\right)$ converges to $\bar{Q} \circ \bar{P}(z)$. Thus, by (5), the property (b) holds for $\bar{Q} \circ \bar{P}$ if and only if $\bar{B}\left(\bar{P}(z), \bar{A}\left(x_{\mu}, z\right)\right)$ converges weak-star to $\bar{Q} \circ \bar{P}(z)$ for every net $\left(x_{\mu}\right) \subset X$ converging weak-star to $z \in X^{* *}$.

In Proposition 2.3 we have shown, roughly speaking, that if the "size" of the image of $\bar{P}$ is "small", then the equality $\overline{Q \circ P}=\bar{Q} \circ \bar{P}$ holds even if the middle space $Y$ is not symmetrically regular. The next example shows that even in the case $Z=\mathrm{C}$ we can find $P$ and $Q$ such that $\overline{Q \circ P} \neq \bar{Q} \circ \bar{P}$.

Example 2.6. Define the bounded symmetric bilinear mappings $A: l_{1} \times$ $l_{1} \rightarrow l_{1}$ by

$$
\begin{aligned}
A(x, y)=\sum_{n=1}^{\infty}\left[\left(x_{1} e_{1}+x_{3} e_{3}+\cdots+x_{2 n-1} e_{2 n-1}\right) y_{2 n}\right. \\
\left.\quad+\left(y_{1} e_{1}+y_{3} e_{3}+\cdots+y_{2 n-1} e_{2 n-1}\right) x_{2 n}\right] \\
+\sum_{n=1}^{\infty}\left[\left(x_{1}+x_{3}+\cdots+x_{2 n-1}\right) y_{2 n}\right. \\
\left.\quad+\left(y_{1}+y_{3}+\cdots+y_{2 n-1}\right) x_{2 n}\right] e_{2 n},
\end{aligned}
$$


and $B: l_{1} \times l_{1} \rightarrow \mathrm{C}$

$$
B(x, y)=\sum_{n=1}^{\infty}\left(x_{1}+x_{3}+\cdots+x_{2 n-1}\right) y_{2 n}+\left(y_{1}+y_{3}+\cdots+y_{2 n-1}\right) x_{2 n},
$$

where $x=\left(x_{i}\right), y=\left(y_{i}\right) \in l_{1}$ and $\left\{e_{n}\right\}$ is the canonical basis of $l_{1}$. Let $P$ and $Q$ be the 2-homogeneous polynomials from $l_{1}$ to $l_{1}$ associated to $A$ and $B$, respectively. Then $\overline{Q \circ P} \neq \bar{Q} \circ \bar{P}$.

Proof. Clearly

$$
B\left(e_{2 p}, e_{2 q}\right)=0, \quad B\left(e_{2 p-1}, e_{2 q-1}\right)=0
$$

for every positive integers $p, q$. Further, we obtain that

$$
B\left(e_{2 p}, e_{2 q-1}\right)= \begin{cases}1 & \text { if } p \geq q \\ 0 & \text { if } p<q .\end{cases}
$$

Let $\alpha$ and $\beta$ be weak-star limit points in $\ell_{l}^{* *} \backslash \ell_{l}$ of the sets $\left\{e_{2 k-1}: k \in \mathbf{N}\right\}$ and $\left\{e_{2 k}: k \in \mathbf{N}\right\}$, respectively. It follows immediately from the above that

$$
\bar{B}\left(e_{2 q-1}, \alpha\right)=\bar{B}\left(e_{2 p}, \alpha\right)=\bar{B}\left(e_{2 p}, \beta\right)=0, \quad \bar{B}\left(e_{2 q-1}, \beta\right)=1
$$

for every positive integers $p$ and $q$. By taking limits we have that

$$
\bar{B}(\alpha, \alpha)=\bar{B}(\beta, \beta)=\bar{B}(\beta, \alpha)=0, \quad \bar{B}(\alpha, \beta)=1 .
$$

Hence

$$
\bar{Q}(\alpha+\beta)=\bar{B}(\alpha+\beta, \alpha+\beta)=1, \quad \bar{B}\left(\alpha+\beta, e_{2 q-1}+\beta\right)=2,
$$

for every positive integer $q$.

Therefore, combining (4) and (6) we have that

$$
(\bar{Q} \circ \bar{P})(\alpha+\beta)=\bar{Q}(\alpha+\beta)=1
$$

and

$$
\bar{B}\left(\bar{P}(\alpha+\beta), \bar{A}\left(e_{2 q-1}+e_{2 p}, \alpha+\beta\right)\right)=2,
$$

for every positive integers $p$ and $q$. Hence if $\left(x_{\mu}\right)$ is a net in $X$ converging weak-star to $(\alpha+\beta)$ such that each $x_{\mu}$ is of the form $e_{2 q-1}+e_{2 p}$ we have that $\bar{B}\left(\bar{P}(\alpha+\beta), \bar{A}\left(x_{\mu}, \alpha+\beta\right)\right)$ does not converge to $(\bar{Q} \circ \bar{P})(\alpha+\beta)$. By Lemma 2.5 we obtain that $\overline{Q \circ P} \neq \bar{Q} \circ \bar{P}$.

It is possible in the above example to proceed as in Example 2.4 to obtain that $\overline{Q \circ P}(\alpha+\beta)=1$ but $\bar{Q} \circ \bar{P}(\alpha+\beta)=\frac{5}{3}$. 


\section{Numerical range of a holomorphic mapping}

Let $T$ be a bounded linear operator from a complex Banach space $X$ into $X$. The numerical range of $T$ is defined as

$$
V(T)=\left\{\phi(T x): x \in S_{X}, \phi \in S_{X^{*}}, \phi(x)=1\right\},
$$

where $S_{X}$ denotes the unit sphere of $X$ ([6]). The numerical range for a holomorphic mapping was introduced by L. Harris [13]. We define the numerical range of $f \in \mathscr{H}_{b}(X, X)$ to be the set

$$
V(f)=\left\{\phi(f(x)): x \in S_{X}, \phi \in S_{X^{*}}, \phi(x)=1\right\} .
$$

The numerical ranges of multilinear mappings and polynomials have also been studied since 1996 ([1], [9]).

Bollobás [5] showed that $\operatorname{cl}(V(T))=\operatorname{cl}\left(V\left(T^{*}\right)\right)$, where $T^{*}$ is the adjoint of $T$ and $\operatorname{cl}(S)$ is the norm closure of the subset $S$ of $X$. In the following we will prove that $\operatorname{cl}(V(f))=\operatorname{cl}(V(\bar{f}))$ for $f \in \mathscr{H}_{b}(X, X)$.

THEOREM 3.1. $\operatorname{cl}(V(f))=\operatorname{cl}(V(\bar{f}))$ for $f \in \mathscr{H}_{b}(X, X)$.

Proof. Without loss of generality, we may assume that $\sup _{x \in B_{X}}\|f(x)\| \leq$ 1. It is obvious that $\operatorname{cl}(V(f)) \subset \operatorname{cl}(V(\bar{f}))$. Thus it suffices to show that $V(\bar{f}) \subset$ $\operatorname{cl}(V(f))$.

Suppose that $z \in S_{X^{* *}}, \Psi \in S_{X^{* * *}}$ and $\Psi(z)=1$. Hence $\Psi(\bar{f}(z)) \in$ $V(\bar{f})$. By [10, Theorem 1], there is a net $\left(x_{\alpha}\right) \subset B_{X}$ such that $\left(x_{\alpha}\right)$ converges polynomial-star to $z$ (i.e., $\left(P\left(x_{\alpha}\right)\right)$ converges to $\bar{P}(z)$ for all scalar valued bounded polynomial $P$ on $X)$. Since

$$
\lim \inf \left\|x_{\alpha}\right\| \geq \lim _{\alpha}\left|\phi\left(x_{\alpha}\right)\right|=|\bar{\phi}(z)|=|z(\phi)|
$$

for all $\phi \in S_{X^{*}}$, we have that $\lim _{\alpha}\left\|x_{\alpha}\right\|=1$. Set $y_{\alpha}=\frac{x_{\alpha}}{\left\|x_{\alpha}\right\|}$. Since

$$
\lim _{\alpha} Q\left(y_{\alpha}\right)=\lim _{\alpha} \frac{1}{\left\|x_{\alpha}\right\|^{k}} Q\left(x_{\alpha}\right)=\bar{Q}(z)
$$

for every $Q \in \mathscr{P}\left({ }^{k} X\right)$ and every positive integer $k$, the net $\left(y_{\alpha}\right)$ converges polynomial-star to $z$.

Let $\varepsilon>0$ be given. Since $f$ is uniformly continuous on $B_{X}$, there exists $\delta>0$ such that $\|f(x)-f(y)\| \leq \frac{\varepsilon}{3}$ if $\|x-y\| \leq \delta$ and $x, y \in B_{X}$. Choose $0<\varepsilon_{0}<\frac{1}{2}$ so that $\varepsilon_{0}+\varepsilon_{0}^{2}<\delta$, and $3 \varepsilon_{0} \leq \epsilon$. As $B_{X^{*}}$ is $w\left(X^{* * *}, X^{* *}\right)$-dense in $B_{X^{* * *}}$, considering two elements $z$ and $\bar{f}(z)$ in $X^{* *}$ there exists $\varphi \in B_{X^{*}}$ such that

$$
|\bar{\varphi}(z)-\Psi(z)|=|\bar{\varphi}(z)-1|<\frac{\varepsilon_{0}^{2}}{4}
$$


and

$$
|\bar{\varphi}(\bar{f}(z))-\Psi(\bar{f}(z))|<\frac{\varepsilon_{0}^{2}}{12},
$$

which implies that $1-\frac{\varepsilon_{0}^{2}}{4}<\|\varphi\| \leq 1$. Set $\psi=\frac{\varphi}{\|\varphi\|}$. We have

$$
\begin{aligned}
|\bar{\psi}(z)-1|=\left|\frac{\bar{\varphi}}{\|\varphi\|}(z)-1\right| & \leq\left|\frac{\bar{\varphi}}{\|\varphi\|}(z)-\bar{\varphi}(z)\right|+|\bar{\varphi}(z)-1| \\
& \leq(1-\|\varphi\|)+\frac{\varepsilon_{0}^{2}}{4}<\frac{\varepsilon_{0}^{2}}{2},
\end{aligned}
$$

and similarly,

$$
|\Psi(\bar{f}(z))-\bar{\psi}(\bar{f}(z))|<\frac{\varepsilon_{0}}{3} .
$$

As $\left(y_{\alpha}\right)$ converges polynomial-star to $z$, we have that

$$
1-\psi\left(y_{\alpha}\right) \rightarrow 1-\bar{\psi}(z) \quad \text { and } \quad \psi \circ f\left(y_{\alpha}\right) \rightarrow \overline{\psi \circ f}(z) .
$$

Hence we can choose $y_{0}:=y_{\alpha_{0}}$ such that

$$
\left|\overline{\psi \circ f}(z)-\psi\left(f\left(y_{0}\right)\right)\right|<\varepsilon_{0} / 3 \quad \text { and } \quad\left|1-\psi\left(y_{0}\right)\right|<\varepsilon_{0}^{2} / 2 .
$$

By [5, Theorem 1], there exist $y \in S_{X}$ and $\phi \in S_{X^{*}}$ such that $\phi(y)=1$, $\|\psi-\phi\|<\varepsilon_{0}$ and $\left\|y-y_{0}\right\|<\varepsilon_{0}+\varepsilon_{0}^{2}$. By the construction of the AronBerner extension $\bar{f}$ it is easily checked that $\overline{\psi \circ f}=\bar{\psi} \circ \bar{f}$, and it follows that

$$
\begin{aligned}
& |\Psi(\bar{f}(z))-\phi(f(y))| \\
& \leq|\Psi(\bar{f}(z))-\bar{\psi}(\bar{f}(z))|+\left|\bar{\psi}(\bar{f}(z))-\psi\left(f\left(y_{0}\right)\right)\right| \\
& \quad+\left|\psi\left(f\left(y_{0}\right)\right)-\phi\left(f\left(y_{0}\right)\right)\right|+\left|\phi\left(f\left(y_{0}\right)\right)-\phi(f(y))\right| \\
& \leq \frac{\varepsilon_{0}}{3}+\frac{\varepsilon_{0}}{3}+\|\psi-\phi\|\left\|f\left(y_{0}\right)\right\|+\|\phi\|\left\|f\left(y_{0}\right)-f(y)\right\| \\
& \leq \frac{2}{3} \varepsilon_{0}+\varepsilon_{0}+\frac{\varepsilon}{3}<\varepsilon,
\end{aligned}
$$

which implies that $\Psi(\bar{f}(z)) \in \operatorname{cl}(V(f))$, because $\phi(f(y)) \in V(f)$.

Corollary $3.2\left(\left[8\right.\right.$, Corollary 2.14]). Let $P \in \mathscr{P}\left({ }^{m} X, X\right)$. Then $\operatorname{cl}(V(\bar{P}))$ $=\operatorname{cl}(V(P))$, where $\bar{P}$ denotes the Aron-Berner extension of $P$.

During the preparation of an earlier draft of this paper we became aware that in [1, Lemma 3] the above corollary had been proved for the case $P(x)=$ $x_{1}^{*}(x) \ldots x_{m}^{*}(x)$, where $x_{j}^{*} \in X^{*}, j=1, \ldots, m$. We also want to thank María 
Acosta for pointing out a mistake that we had in the proof of Theorem 3.1 in that former draft.

\section{REFERENCES}

1. Acosta, M. D., Guerrero, J. B., and Galán, M. R., Numerical radius attaining polynomials, Quarterly J. Math. 54(1) (2003), 1-10.

2. Aron, R. M., and Berner, P. D., A Hahn-Banach extension theorem for analytic mappings, Bull. Soc. Math. France 106 (1978), 3-24.

3. Aron, R. M., Cole, B. J., and Gamelin, T. W., Spectra of algebras of analytic functions on a Banach space, J. Reine Angew. Math. 415 (1991), 51-93.

4. Aron, R. M., Schottenlocher, M., Compact holomorphic mappings on Banach spaces and the approximation property, J. Funct. Anal. 21 (1976), 7-30.

5. Bollobás, B., An extension to the theorem of Bishop and Phelps, Bull. London Math. Soc. 2 (1970), 181-182.

6. Bonsall, F. F., and Duncan, J., Numerical Ranges of Operators on Normed Spaces and of Elements of Normed Algebras, London Math. Soc. Lecture Note Ser. (1971).

7. Carando, D., Extendible polynomials on Banach spaces, J. Math. Anal. Appl. 233 (1999), 359-372.

8. Choi, Y. S., García, D., Kim, S. G., and Maestre, M., The polynomial numerical index of a Banach space, Proc. Edinburgh Math. Soc. 49 (2006), 32-52.

9. Choi, Y. S., and Kim, S. G., Norm or numerical radius attaining multilinear mappings and polynomials, J. London Math. Soc. (2) 54 (1996), 135-147.

10. Davie, A. M., and Gamelin, T. W., A theorem on polynomial-star approximation, Proc. Amer. Math. Soc. 106 (1989), 351-356.

11. Harris, L. A., Schwarz lemma in normed linear spaces, Proc. Nat. Acad. Sci. U.S.A. 62 (1969), 1014-1017.

12. Harris, L. A., A continuous form of Schwarz's lemma in normed linear spaces, Pacific J. Math. 38 (1971), 635-639.

13. Harris, L. A., The numerical range of holomorphic functions in Banach spaces, Amer. J. Math. 93 (1971), 1005-1019.

14. Lumer, G., Semi-inner-product spaces, Trans. Amer. Math. Soc. 100 (1961), 29-43.

15. Zalduendo, I., A canonical extension for analytic functions on Banach spaces, Trans. Amer. Math. Soc. 320 (1990), 747-763.

DEPARTMENT OF MATHEMATICS

POSTECH

POHANG (790-784)

KOREA

E-mail: mathchoi@postech.ac.kr
DEPARTAMENTO DE ANÁLISIS MATEMÁTICO UNIVERSIDAD DE VALENCIA

DOCTOR MOLINER 50

46100 BURJASOT (VALENCIA)

SPAIN

E-mail: domingo.garcia@uv.es, manuel.maestre@uv.es

DEPARTMENT OF MATHEMATICS

KYUNGPOOK NATIONAL UNIVERSITY

TAEGU (702-701)

KOREA

E-mail: sgk317@knu.ac.kr 